\title{
Experimental Validation of an Integrated Controls-Structures Design Methodology
}

\author{
Peiman G. Maghami \\ Sandeep Gupta \\ Kenny B. Elliot \\ Joseph E. Walz \\ NASA Langley Research Center \\ Hampton, Virginia 23681
}

\begin{abstract}
The first experimental validation of an integrated controls-structures design methodology for a class of large order, flexible space structures is described. Integrated redesign of the controls-structures-interaction evolutionary model, a laboratory testbed at NASA Langley, was described earlier. The redesigned structure was fabricated, assembled in the laboratory, and experimentally tested against the original structure. Experimental results indicate that the structure redesigned using the integrated design methodology requires significantly less average control power than the nominal structure with control-optimized designs, while maintaining the required line-of-sight pointing performance. Thus, the superiority of the integrated design methodology over the conventional design approach is experimentally demonstrated. Furthermore, amenability of the integrated design structure to other control strategies is evaluated, both analytically and experimentally. Using Linear-QuadraticGuassian optimal dissipative controllers, it is observed that the redesigned structure leads to significantly improved performance with alternate controllers as well.
\end{abstract}




\section{Introduction}

The need and benefits of controls-structures integrated design of high precision flexible strucutres is well recognized in the literature ${ }^{1-7}$. Many numerical studies have been conducted to demonstrate the benefits of these design approaches ${ }^{1-6}$. However, experimental validation of these benefits with realistic models of large-order, flexible space structures has been lacking. One of the goals of the controls-structures interaction (CSI) program at NASA Langley ${ }^{3}$ has been to develop and validate integrated controls-structures design methodolgy for various classes of flexible spacecraft. To accomplish this goal, an integrated controls-structures design methodology was developed and applied to a redesign of a realistic flexible test structure at NASA Langley, as described in Ref. 6. The primary thrust of the current effort was to verify by experiment, advantages of the integrated design methodology, as predicted by analytical studies in Ref. 6. This paper presents the first experimental validation of the benefits of integrated design by fabrication and testing of the redesigned CSI evolutionary model (CEM).

The phase-0 CSI CEM is a ground-based flexible test structure at NASA Langley, shown in Fig. 1. The structure consists of a long main bus, a laser tower, a reflector tower, and two horizontal booms for suspension of the structure. A laser beam from a source mounted at the top of the laser tower is reflected off a mirrored surface on the reflector tower, on to a detector surface on the ceiling. As the structure vibrates, the deviation of laser point on the detector surface from its nominal location is used as a measure for error in line-of-sight pointing for this system. Bi-directional thrusters are used as control actuators and for disturbance injection; and collocated accelerometers are used for feedback control measurements. Integrated redesign of this structure with respect to 
both structural and control parameters was described in Ref. 6. The design objective was to minimize average control power required to maintain line-of-sight pointing deviations within specified limits. As described in Ref. 6, redesign of the CSI CEM structure with static dissipative compensators resulted in a $40 \%$ reduction in control power, whereas the redesign with dynamic dissipative compensators produced a $44 \%$ reduction in control power. Structural designs from both these integrated redesigns demonstrated similar mass and stiffness distributions. Ultimately, a structural design which was close to both these designs was selected for fabrication and assembly in the laboratory, and is referred to as the phase-1 CEM structure. It should be noted that in Ref. 6 the structural designs were obtained with the aid of design guides to ensure that the resulting structural design using the integrated controls-structres approach could be fabricated in a cost effective manner. A description of the experimental setup and validation of the analytically predicted benefits from this integrated redesign is presented in the following sections.

One consideration for the design of the controller and the structure in a unified environment is that the control law must be specified at the preliminary design stage. However, it may not be feasible to specify the control law without prior experimental identification of the dynamics of the structural system. This brings about the issue of the sensitivity of the designed structure to a specific control architecture used in the integrated design. In order to investigate this sensitivity, control-optimized designs are performed on both the phase-0 and phase-1 CEM structures, using LQG optimal dissipative compensators, which was not used in the integrated redesign of the phase-1 CEM. An analytical as well as experimental comparison of optimal control designs with LQG optimal dissipative compensators is presented. It is seen that the benefits of the integrated design approach, 
in terms of significant reduction in the average control power required, carry over to this alternate controller as well.

In section II, control-optimized designs of LQG optimal dissipative controllers for both phase-0 CEM and phase-1 CEM are presented, alongwith an analytical comparison of the power required for maintaining specified line-of-sight. Then, in section III, a brief description of the experimental setup is presented, followed by the experimental validation results and discussions in section IV.

\section{Evaluation of an Alternative Controller}

In order to investigate the sensitivity of the integrated design to changes in the control algorithm, LQG optimal dissipative controllers are used in place of static and dynamic dissipative controllers, which were used in the integrated redesign of the phase0 CEM. A parameterization of these controllers is presented in this section for synthesis of control-optimized compensators for both phase-0 and phase-1 CEM structures. An analytical comparison of the control power required to maintain line-of-sight errors within specified limits, is performed, similar to the comparisons for static and dynamic dissipative compensators in Ref. 6.

LQG design methods have been popular in synthesizing multivariable feedback controllers for multi-input, multi-output plants. Although stability of the nominal closed loop is guaranteed by LQG theory, closed-loop stability cannot be guaranteed in the presence of unmodelled dynamics and parametric uncertainties, which are commonly associated with

flexible space structures. However, if the LQG compensator is restricted to be dissipative, then closed loop stability is guaranteed from passivity considerations ${ }^{2}$. The constraints on 
the LQG compensator design matrices which lead to a dissipative compensator have been developed in Ref. 8, and are summarized below.

For the linear time-invariant system

$$
\begin{aligned}
& \dot{x}=A x+B u+v \\
& y=C x+w
\end{aligned}
$$

the LQG optimal compensator is given by

$$
\begin{aligned}
& \dot{\hat{x}}=\left(A-B R^{-1} B^{T} P_{c}-P_{f} C^{T} W^{-1} C\right) \hat{x}+P_{f} C^{T} W^{-1} y \\
& u=-R^{-1} B^{T} P_{c} \hat{x}
\end{aligned}
$$

where $P_{c}=P_{c}^{T}>0$ and $P_{f}=P_{f}^{T}>0$ are solutions of the following Riccati equations

$$
\begin{gathered}
A^{T} P_{c}+P_{c} A-P_{c} B R^{-1} B^{T} P_{c}+Q=0, \\
P_{f} A^{T}+A P_{f}-P_{f} C^{T} W^{-1} C P_{f}+V=0,
\end{gathered}
$$

with $Q=Q^{T} \geq 0$ and $R=R^{T}>0$ being the weighting matrices, and $V=V^{T} \geq 0$, $W=W^{T}>0$ being the noise covariance matrices. The matrices $W$ and $V$ are used as design parameters rather than noise covariances, and have to satisfy certain constraints, specified below, for the resulting compensator to be positive real. If the linear system of Eq. 1 is passive, that is, its transfer function is positive real, the system matrices satisfy the following conditions of the positive realness lemma ${ }^{2}$ : there exists a symmetric positive definite matrix, $P=P^{T}>0$, and a symmetric positive semidefinite matrix, $\hat{Q}=\hat{Q}^{T} \geq 0$, which satisfy the following conditions,

$$
\begin{array}{r}
A^{T} P+P A=-\hat{Q}, \\
P B=C^{T} .
\end{array}
$$


If the matrices $V$ and $W$ are constructed as

$$
\begin{aligned}
& V=P^{-1} \hat{Q} P^{-1}+B R^{-1} B^{T}, \\
& W=R
\end{aligned}
$$

where the weighting matrix $R=R^{T}>0$ is an arbitrary, symmetric positive definite matrix, and the weighting matrix $Q$ is a symmetric positive definite matrix satisfying

$$
Q-P B R^{-1} B^{T} P \equiv Q_{1}>0
$$

where $Q_{1}=Q_{1}^{T}>0$ is an arbitrary, symmetric positive definite matrix, then the compensator in Eq. 2 is dissipative ${ }^{8,9}$. The dissipative nature of the compensator guarantees closed loop stability in the presence of unmodelled dynamics and parametric uncertainties of the open-loop plant model.

Control-optimized LQG-type dissipative compensators were designed and experimentally tested for both phase-0 CEM and phase-1 CEM structures. The parameters used for synthesis of dissipative LQG compensators as follows. The matrices

$$
\hat{Q}=2 \operatorname{diag}\left(0, \zeta_{1} \omega_{1}, \ldots, 0, \zeta_{r} \omega_{r}\right)
$$

and

$$
P=\operatorname{diag}\left(\omega_{1}^{2}, 1, \ldots, \omega_{r}^{2}, 1\right)
$$

with the system matrices for the structural plant in modal form, as in Ref. 6, satisfy the conditions for positive realness in Eq. 4. The matrices $R=R^{T}>0$ and $Q_{1}=$ $Q_{1}^{T}>0$ were chosen to be diagonal matrices, and their elements were used as the control design variables to obtain control-optimized designs. The dissipative LQG compensator is essentially a dynamic dissipative controller, represented in state space form as

$$
\dot{x}_{c}=A_{c} x_{c}+B_{c} y, \quad u=-C_{c} x_{c}
$$


with Thus, the closed-loop equations are the same as those for dynamic dissipative compensators in Ref. 6. Computation of the control power required and the root-meansquare line-of-sight error is performed using the state covariance matrix of the closed-loop system, as described in Ref. 6. Interior penalty function techniques are employed to obtain optimal compensator parameters which minimize the control power requirements. The number of modes used in the control design model for controller synthesis depended on the number of significant modes in the input-output characterization of the structure. For the phase-0 CEM structure, a 21-mode model was used to characterize the significant inputoutput properties of the plant model. Thus, the design variables used for the optimal control design are the 42 diagonal elements of $Q_{1}$ followed by 6 diagonal elements of $R$. For control optimized dissipative LQG compensator of the phase-1 CEM, an 18 mode model was used based on the input-output characterization of this structure. The design variables for the optimal controller for phase-1 CEM were 36 diagonal elements of $Q_{1}$ followed by 6 diagonal elements of $R$. The numerical values of the optimal control designs are available in Ref. 7. Figure 2 compares the maximum and minimum singular value plots of LQG dissipative controllers for phase-0 CEM (in dashed lines) and phase-1 CEM (in solid lines). It is observed that the controller gains for phase-1 CEM are smaller than the controller gains for phase-0 CEM throughout the entire frequency spectrum, and the power distribution of the controllers over various frequencies is significantly different for the two structures. Analytical results show that to maintain a root-mean-square line-of-sight pointing error of $2.0 \mathrm{in}$, the phase-0 CEM requires a control power of $5.93 \mathrm{lb}^{2}$, while phase-1 CEM requires only $2.65 \mathrm{lb} b^{2}$. This shows that even though dissipative LQG controllers were not used for integrated design, the performance improvement due to structural redesign alone leads to 
over $45 \%$ reduction in control effort.

Since designs with both static and dynamic dissipative control laws lead to similar mass and stiffness distributions for the structure, it was anticipated that alternate control laws, which were not used for integrated redesign, would also demonstrate enhanced performance in comparison to the original structure. These analytical results presented in this section validate this conjecture for LQG optimal dissipative compensators. The integrated design process resulted in a structure which is more amenable for active control with alternate control design strategies as well.

\section{Hardware Description}

This section describes the experimental setup for the active control experiments with phase-0 CEM and phase-1 CEM structures. A detailed description of this system can be found in Ref. 10. This section reviews some elements of the CEM system which are relevant to the active control experiments performed to validate the benefits of the integrated controls-structures design methodology.

\section{a. Structural System}

A schematic of the CEM structure is shown in Fig. 1, and a photograph of the experimental setup is shown in Fig. 3. The structure consists of a 62 bay long central truss, a 11 bay laser tower, a 6 bay reflector tower, and four 10 bay suspension booms. The bays are cubical with 10 inch sides, and are built from aluminium tubes and node balls with high-strength steel connectors between the node balls and the tubes. The reflector on one end of the structure is 192 inch in diameter, and simulates large antennas and other appendages found on space platforms. The reflector is a ribbed structure with a mirrored 
surface which reflects the laser beam for the line-of-sight pointing subsystem. It is rotated from the horizontal plane such that the laser beam is reflected vertically to a detector system on the ceiling. This structural system is suspended from the ceiling using two primary attach points. Two suspension cables are yoked such that four points on the CEM are connected to the suspension system, as shown in Fig. 1. Linear extension springs are attached to the suspension cables to reduce the effects of rigid-body pendulum modes from the flexible body dynamics of the CEM structure.

\section{b. Laser Scoring System}

A laser motion optical detector system ${ }^{10}$ was used for assessing the line-of-sight performance of the CEM structure. This system uses a 5 milliwatt Helium-Neon laser and a microprocessor scanned photo-diode array to detect linear beam position over a 40 inch by 40 inch detector surface. The laser source is mounted on the top of the laser tower, and the detector surface is on the ceiling directly above the reflector. Low power lasers were used to minimize health hazards, particularly to the eye, but this required that the laboratory be quite dark during the experiments to allow adequate laser beam to background noise intensity ratio for the detector system. The resolution of the detector array is limited by the number of photo-diodes in the detector array and the estimation algorithms used to determine the beam location. For the system used in these experiments, a resolution of about 0.2 inches over the detector area were achieved. The detector system provided two analog signals proportional to the beam displacement from the origin in two perpendicular axes. The position of the laser beam was given in inches by $X=2.23 V_{x}$ and $Y=2.23 V_{y}$, where $V_{x}$ and $V_{y}$ are the analog output voltages ${ }^{10}$. 


\section{c. Sensors and Actuators}

Servo accelerometers were used for measuring linear accelerations, as input to the control algorithms. These accelerometers are placed at eight locations along the directions marked one through eight in Fig. 1. The linear range of these accelerometers was $+/-30$ $g$ with sensitivity of 5 volts/g. The dynamics of these accelerometers was negligible for the desired bandwidth of the active control experiments. For control actuation and disturbance noise injection, bi-directional air thrusters were used. These thrusters were installed in pairs on opposite sides of cubical bays to insure pure translational forces. Each of these thrusters was bench tested to characterize its dynamics. These actuator dynamics were characterized experimentally in the form $f(s)=55 v(s) /(s+273)$, where $f$ is the force in lbs and $v$ is the input voltage in volts ${ }^{10}$. These actuators are almost collocated at the eight control stations shown in Fig. 1. Note that since the thrusters are used in pairs, the effective transfer function at each location becomes $110 /(s+273)$.

\section{d. Data Acquisistion and Processing}

The signal conditioning, data acquisition, and control processing were performed in a control room located next to the testbed. All signals to and from the sensors were analog. Most sensor signals passed through a junction box outside the control room, and then went on to a patch panel. The patch panel allowed the signals to be routed to data acquisition systems, control systems, recorders and/or trouble shooting equipment. Actuator commands from signal generators or the control system were routed though a safety system before being sent to the testbed. The safety system provided for manual as well as automatic (limit checking) shut off capability. Signals from the safety system were sent to the thruster signal 
conditioners located on the CEM structure. The Computer Automated Measurement and Control (CAMAC) system at NASA Langley was used for data acquisition for closed-loop control. The system provided analog-to-digital and digital-to-analog interfaces to several computing platforms. The CAMAC system is composed of modules mounted on a crate, which provided the interfaces between external processes and internal buses called dataways. The crate modules also have an option of providing analog third order Bessel filters at various frequencies. Real-time control computations were performed on a VAX computer from Digital Equipment Corp., or optionally on a CYBER machine. The VAX computer is located in the control room, while data is transferred over $50 \mathrm{Mbit} / \mathrm{sec}$ fiber-optic links to the CYBER machine in a remote location. The system could support real-time controllers at rates upto $200 \mathrm{~Hz}$, with 8 inputs and 11 outputs. Input and output experimental data is stored as Matlab binary file at the completion of the experiment.

\section{e. Structural Strut Elements}

The CEM system is similar for both phase-0 an phase-1 CEM, except the sizes of individual struts for the structure. The effective areas of the struts had been used to characterize the size of the struts, as discussed in Ref. 6 . The effective areas for the battens and longerons of phase- 0 CEM is $0.134 i n^{2}$ and the effective areas for diagonals is 0.124 $i n^{2}$. The structural designs obtained from integrated redesign of the phase-0 CEM structure exhibited similar trends in mass distribution, and the optimal values for structural design variables were within $20 \%$ of each other. Therefore, a structural design which was close to both these designs was selected for fabrication and assembly in the laboratory. The structural elements of the optimal structure, referred to as phase-1 CEM, are given in Table 
1. Note that the production values of the elements for the assembled structure were chosen to approximate the design trends from the numerical studies, but to avoid excessive costs, the number of different size struts was kept as small as possible. In consonance with the design trends, all diagonals were chosen of the same size, and all battens were of the same size. Four different sizes of the longerons were used for the various sections as shown in Table 1. Table 2 presents the modal frequencies of the first ten modes of the fabricated phase-1 CEM and phase-0 CEM ${ }^{10,11}$. A comparison of these frequencies indicate that the first six frequencies associated with the suspended structure have not been changed significantly, mainly because the changes in the structure can affect these frequencies only through changing the center of mass of the structure, and not directly as for the flexible modes. On the other hand, the frequencies of the flexible modes, particularly the second and third flexible modes, have increased considerably (as much as $30 \%$ ). The second flexible mode frequency increased from $1.74 \mathrm{~Hz}$ to $2.25 \mathrm{~Hz}$ and the third flexible mode frequency from $1.88 \mathrm{~Hz}$ to $2.40 \mathrm{~Hz}$, making these modes and the structure less sensitive to disturbances at stations 1 and 2 .

The next section presents the active control experiments performed on the CEM structures. 


\section{Experimental Validation}

Although numerous numerical studies in the literature have demonstrated the benefits of controls-structures integrated design, there has been no experimental validation of these benefits for large-order flexible structures. The primary thrust of this effort was to experimentally verify the advantage of the integrated design methodology as observed in analytical studies. Of course, the paramount difficulty in experimental verification is that some assumptions made in the analytical developments are not necessarily valid in the laboratory. Some of these issues are discussed below.

As mentioned before, dissipative system theory requires that the sensors and actuators be collocated. This collocation was not truly possible, but the sensors and actuators were located so close together (within the bays at the six control stations) that they could be considered to be collocated for all practical purposes. Second, continuous time framework has been used in the dissipative theory to demonstrate guaranteed stability robustness, whereas the implementation in the laboratory was digital. The theoretical framework breaks down with discretization of the continuous time plant and controllers; however, since the sampling rates used were much faster than the control bandwidths considered, the effects of discretization are negligible.

Sensor and actuator dynamics have been ignored in the integrated design. Though there is no appreciable dynamics associated with the accelerometers, the bi-directional air thrusters experimentally exhibited first order dynamics of the form $110 /(s+273)$. However, actuator bandwidth, implied by this dynamics, was much larger than the control bandwidths (in $2-5 \mathrm{~Hz}$ range) considered, so that ignoring the actuator dynamics for integrated redesign was justified. Also, the velocity signals required for the controllers were 
obtained by integrating the accelerometer signals with the aid of wash-out filters. The washout filters were used in order to remove the constant uncertain bias in the accelerometer signal.

White noise disturbances were assumed in integrated design and analysis. However, for the experimental work, band-limited white noise was used because the thrusters have limited power. The bandwidth for the white noise employed was large enough to cover the controller bandwidth, so that for these control experiments the noise may be treated as white noise. Further, integrated design and analysis assumes continuous time white noise, whereas in the experiments the noise was discretized at the sampling rates of the control implementation. The disturbance sequence used for the tests was made as large as possible within the maximum safety limit and the maximum power available from the disturbance actuators. At each time step the noise levels were normally distributed in the discrete-time noise sequence employed for the experiments.

A computer simulation model for the laboratory structure, incorporating all the issues detailed above, was developed using the Matlab software. The mode shapes for the plant model in the simulation were obtained from a detailed NASTRAN model of the structure. System identification tests had been performed to obtain experimental modal frequency and damping values ${ }^{10,11}$. The system identification tests involved significant motion of the structure, which induced additional damping due to the motion of hoses feeding the air thrusters and other such nonlinearities. Therefore, the identified modal damping values are expected to be higher than those encountered in the disturbance rejection experiments, which involved much smaller motion. The plant model for the simulations included modes up to $50 \mathrm{~Hz}$ (about eighty modes), as opposed to about thirty modes used for control 
design, along with the experimentally identified damping values. The compensator system matrices for simulations were exactly those that were loaded in the real-time control software. Finally, the excitation-control scenario and the digital implementation for the simulations was identical to that used in the experiments. The goal was to obtain a simulation of the laboratory experiment such that it could be used to evaluate our ability to predict actual behavior of the test structure.

The test sequence for the active control experiments began with initialization to zero out accelerometer bias and to ensure the structure was at rest for zero initial conditions. Then, the disturbance sequence was introduced at actuator stations 1 and 2. After sixty seconds (about 4-5 time constants of the slowest mode), for the transients to settle down, data was collected for steady state analysis. Time histories of the applied control effort and the line-ofsight deviations obtained from the experiments were analyzed, and compared with computer simulations for each test case. Mean square values for the control effort, and the line-of-sight error vector were computed as the standard deviations of the observed discrete data. Exactly the same experiments were performed on both structures, the nominal phase-0 CEM and the redesigned phase-1 CEM. Results of disturbance rejection experiments performed with static dissipative controllers, dynamic dissipative controllers and LQG optimal dissipative controllers are discussed in the following paragraphs.

The control inputs at station 3, using static dissipative controllers for phase-0 CEM and phase-1 CEM are shown in Figs. 4a and 4b, respectively, for comparison. These figures graphically illustrate lower levels of control effort required for the redesigned structure. The time axis of these figures starts at 60 seconds because the earlier data is ignored for steady state analysis. The digital implementation of the static dissipative controllers is at $200 \mathrm{~Hz}$, 
so 60 seconds of data yields 12000 time steps. Denoting the control input sequence for an experimental run as $u_{i}(k), i=1, \ldots, 6$, and $k=1, \ldots, 12000$, a running average for the control power is computed as

$$
\operatorname{pow}(k)=\frac{1}{(k-1)} \sum_{j=1}^{k}\left\{\sum_{i=1}^{6} u_{i}^{2}(j)\right\}
$$

This running average is plotted for static dissipative controllers in Fig. 7, and Figs. 10 and 12 for dynamic dissipative and LQG optimal dissipative controllers, respectively.

The deviation of the laser point from its nominal position on the detector system, in the local $x$-coordinate, is presented in Fig. 5 for the static dissipative controller. Subplots $a$ and $b$ of Fig. 5 are for phase-0 CEM and phase- 1 CEM, respectively. The magnitudes are seen to be equal for both systems. Denoting deviations of the laser point from its nominal location on the detector system in local $x$ - and $y$-directions as $x(k)$ and $y(k)$, for $k=1, \ldots, 12000$, a running RMS line-of-sight error is computed as the standard deviation of the data, as follows

$$
\operatorname{rms}(k)=\left(\frac{1}{(k-1)} \sum_{j=1}^{k}\left[x^{2}(j)+y^{2}(j)\right]\right)^{\frac{1}{2}}
$$

The RMS line-of-sight pointing error is plotted for the static dissipative controllers in Fig. 6. Figs. 9 and 11 show these plots for dynamic dissipative and dissipative LQG controllers, respectively. Due to the relatively higher order of the LQG optimal dissipative controllers, the sampling rate for experiments with these controllers had to be reduced to $125 \mathrm{~Hz}$.

The experimental results for static dissipative controllers are shown in Figs. 4 through 7. A comparison of the control inputs at station 3 is shown in Fig. 4, with phase- 0 control input in subplot $a$ and phase-1 CEM control input in subplot $b$. Smaller magnitudes of 
the control effort for phase-1 CEM structure should be noted in Fig. 4. Similar trends are observed for inputs at other control stations as well. Deviations of the laser beam in $x$-direction are of approximately equal magnitude for both phase-0 CEM and phase-1 CEM, as seen in Fig. 5. These levels are similar in the $y$-direction as well. Figure 6 shows that the root-mean-square line-of-sight error for both structures is approximately equal to 0.6 in. Note that the experimentally observed value of RMS pointing error is 0.6 in instead of the analytically computed value of 2.4 in since discrete time, band-limited noise was used for the disturbance input at actuators 1 and 2 in the experiments, whereas the analytical work assumed continuous time white-noise. The difference in RMS pointing error values for phase-0 CEM and phase-1 CEM can be attributed to resolution of the laser detector system in the laboratory, which is $0.2 \mathrm{in}$. Figure 7 illustrates the average control power needed to maintain this line-of-sight pointing error. The control power in the experiment for phase-0 CEM was nearly 2.0 volt $^{2}$, while that for phase-1 CEM is 0.66 volt $^{2}$, a reduction of about $67 \%$. The simulations for these experimental runs show a decrease in control effort from 1.56 volt $^{2}$ for phase-0 CEM to 0.73 volt ${ }^{2}$ for phase-1 CEM (reduction of about $53 \%$ ), which is closer to that predicted by the analysis. However, the savings in control power observed experimentally were more than that predicted by analysis. It is observed from Fig. 7 that the control power level predicted by simulation for the phase-1 CEM matches the experimental level quite well. However, this is not the case for phase-0 CEM. In fact the control power level computed by simulations is about $28 \%$ off from its experimental counterpart. This may be attributed to the spillover excitation of the modes outside the control bandwidth, which have not been parametrized accurately, and other modelling errors ${ }^{10,11}$.

Figures 8 through 10 display the results for experiments with dynamic dissipative 
controllers. Figure 8 compares the control input at station 3 for the two structures, showing the lower control effort for phase-1 CEM structure. The deviations of the laser from its nominal position in $x$ - and $y$ - directions have the same levels. The experimental verification of the advantages of integrated design, for dynamic dissipative controllers, are observed in Figs. 9 and 10. As seen in Figure 9, the RMS line-of-sight pointing error is maintained near 0.6 in for both structures, and the simulation results are consistent. Average control

power of 1.65 volt $^{2}$ for phase-0 CEM was reduced to 0.64 volts ${ }^{2}$ for phase-1 CEM, a reduction of $61 \%$ (see Fig. 10). The simulations show the reduction of control power from 1.35 volts $^{2}$ for phase-0 CEM to 0.7 volts $^{2}$ for phase-1 CEM, (reduction of about 48\%) which is closer to the analytical predictions. Again, the difference between the experimental and simulation predictions may be attributed to the spillover effects from the unmodelled dynamics of the flexible structure. It is also observed by comparing Fig. 7 and Fig. 10 that dynamic dissipative controllers needed less control power than the static dissipative to maintain the same line-of-sight pointing performance for both structures, as predicted by analytical results ${ }^{6}$.

Therefore, it is observed from the experimental results that (a) the benefits of integrated design predicted analytically are achieved experimentally, and that (b) the integrated control-structure designs can provide an overall design which is significantly superior to that obtained through the conventional design approach, requiring much less control power to achieve the same pointing performance.

\section{Alternate Controller}

The results of the disturbance rejection experiments with control-optimized dissipative 
LQG controllers are given in Figs. 11 and 12. Similar to the static and dynamic dissipative results, from a comparison of the control power for phase-0 CEM and phase-1 CEM (Fig. 12), it is observed that phase-1 CEM requires less control power to maintain allowable RMS pointing error. To maintain the RMS line-of-sight at $0.5 \mathrm{in}$, the control power needed for phase- 0 CEM was 1.16 volts ${ }^{2}$, which is reduced to 0.44 volts ${ }^{2}$ for phase- 1 CEM. The reduction in control power is about $62 \%$. In simulations, the control power was reduced from

0.94 volts $s^{2}$ for phase- 0 CEM to 0.36 volts $s^{2}$ for phase-1 CEM, a reduction of about $62 \%$.

These experiments confirm the observation from the analysis in Section II, that the integrated design structure, phase-1 CEM, requires considerably less control power with dissipative LQG controllers as opposed to phase-0 CEM, even though the integrated design process employed static and dynamic dissipative control strategies. Thus, it may be concluded that along with optimizing control power with the selected control design strategy, the integrated design process makes the resulting structure more amenable to control with alternate strategies as well.

\section{Concluding Remarks}

Experimental validation of an optimization-based integrated controls-structures design approach has been presented using two types of dissipative controllers. The nominal phase-0 CEM structure was redesigned to minimize the average control power required to maintain specified rms line-of-sight pointing error under persistent disturbances. The redesigned structure, phase-1 CEM, was assembled in the laboratory and tested against the original structure, phase-0 CEM. Using two different dissipative controllers, namely, static dissipative controller and dynamic dissipative controller, over $60 \%$ reduction in control 
power has been shown experimentally, for maintaining the same line-of-sight pointing performance. Therefore, it has been demonstrated, both analytically and experimentally, that integrated controls-structures design can yield designs which are substantially superior to those obtained through the traditional sequential approach. Although there are numerous analytical and numerical studies in the literature suggesting benefits derived from controlsstructures integrated design, this is the first experimental verification of such performance enhancements for such large-order flexible structures. Moreover, experiments with alternate LQG optimal dissipative controllers, which were not used for integrated redesign, indicate that the integrated design process has a tendency to make the structure more amenable to active control and less excitable by the disturbances, such that superior overall performance may be realized for alternate control design strategies as well.

\section{References}

1. Ketner, G. L., "Survey of Historical Incidences With Controls-Structures Interaction and Recommended Technology Improvements Needed to Put Hardware in Space," Battelle, Pacific Northwest Laboratory, Report No. PNL-6846, Richland, WA, March 1989.

2. Joshi, S. M., Control of Large Flexible Space Structures, edited by M. Thoma and A. Wyner, Volume 131, Lecture Notes in Control and Information Sciences, Springer-Verlag, Berlin, 1989.

3. Newsom, J. R., Layman, W. E., Waites,H. B., and Hayduk, R. J., "The NASA ControlsStructures Interaction Technology Program," NASA Langley Research Center, TM102752, Hampton, VA, October 1990.

4. Rao, S. S., and Pan, T. S., "Robustness Improvement of Actively Controlled Structures Through Integrated Structural/Control Design," Flgiht Dynamics Directorate, Wright Laboratory, Report No. WL-TR-91-3058, Wright-Patterson Air Force Base, OH, May 1991.

5. Maghami, P. G., Joshi, S. M., Armstrong, E. S., and Walz, J. E., "Integrated ControlsStructures Design Methodology Development for a Class of Flexible Spacecraft," Third 
Air Force/NASA Symposium on Recent Advances in Multi-disciplinary Analysis and Optimization, San Fransisco, CA, September 1990, pp. 1-6.

6. Maghami, P. G., Gupta, S., Elliot, K. B., and Joshi, S. M., "Integrated ControlsStructures Design Methodology: Redesign of an Evolutionary Test Structure," Journal of Guidance, Control, and Dynamics, Vol. 19, No. 2, 1996, pp. 316-323.

7. Maghami, P. G., Gupta, S., Elliot, K. B., Joshi, S. M., and Walz, J. E., "Experimental Validation of an Integrated Controls-Structures Design Methodology for a Class of Flexible Space Structures," NASA Langley Research Center, TP-3462, Hampton, VA, November 1994.

8. Lozano-Leal, R., and Joshi, S. M., "On the Design of Dissipative LQG-Type Controllers", Recent Advances in Robust Control, edited by P. Dorato and R. Yedavalli, IEEE Press, New York NY, 1990, pp. 251-252.

9. Joshi, S. M., and Maghami, P. G., "Robust Dissipative Compensators for Flexible Spacecraft Control", IEEE Transactions on Aerospace and Electronic Systems, Vol. 28, No. 3, July 1992, pp. 768-774.

10. Belvin, W. K., Elliot, K. B., Horta, L. G., Bailey, J. P., Bruner, A. M., Sulla, J. L, Won, J., and Ugoletti, R. M., "Langley's CSI Evolutionary Model: Phase 0," NASA Langley Research Center, TM 104165, Hampton, VA, November 1991.

11. Gronet, M. J., et al., "Design, Analysis, and Testing of the Phase 1 CSI Evolutionary Model Erectable Truss", NASA CR-4461, NASA Langley Research Center, Hampton, VA, August 1992. 
Table 1 Effective areas of struts for fabricated Phase-1 CEM.

\begin{tabular}{|c|c|c|c|c|c|c|c|}
\hline $\begin{array}{l}\text { Var. } \\
\text { No. }\end{array}$ & $\begin{array}{c}\text { Longerons, } \\
\text { in^2 }\end{array}$ & $\begin{array}{l}\text { Var. } \\
\text { No. }\end{array}$ & $\begin{array}{l}\text { Battens, } \\
\text { in^2 }\end{array}$ & $\begin{array}{l}\text { Var. } \\
\text { No. }\end{array}$ & $\begin{array}{c}\text { Diagonals, } \\
\text { in^2 }\end{array}$ & $\begin{array}{c}\text { Section } \\
\text { No. }\end{array}$ & Description \\
\hline 1 & 0.333 & 2 & 0.097 & 3 & 0.083 & 1 & main truss \\
\hline 4 & 0.099 & 5 & 0.097 & 6 & 0.083 & 2 & $\begin{array}{l}\text { horizontal } \\
\text { boom }\end{array}$ \\
\hline 7 & 0.175 & 8 & 0.097 & 9 & 0.083 & 3 & laser tower \\
\hline 10 & 0.264 & 11 & 0.097 & 12 & 0.083 & 4 & main truss \\
\hline 13 & 0.264 & 14 & 0.097 & 15 & 0.083 & 5 & main truss \\
\hline 16 & 0.099 & 17 & 0.097 & 18 & 0.083 & 6 & $\begin{array}{l}\text { horizontal } \\
\text { boom }\end{array}$ \\
\hline 19 & 0.099 & 20 & 0.097 & 21 & 0.083 & 7 & $\begin{array}{l}\text { reflector } \\
\text { tower }\end{array}$ \\
\hline
\end{tabular}


Table 2 Comparison of modal frequencies for the fabricated phase-1 CEM and phase-0 CEM.

\begin{tabular}{ccc}
\hline \hline $\begin{array}{c}\text { Mode } \\
\text { No. }\end{array}$ & $\begin{array}{c}\text { Phase-1 } \\
\text { CEM } \\
(\mathbf{H z})\end{array}$ & $\begin{array}{c}\text { Phase-0 } \\
\text { CEM } \\
(\mathbf{H z})\end{array}$ \\
\hline 1 & 0.1475 & 0.1471 \\
2 & 0.1495 & 0.1491 \\
3 & 0.1553 & 0.1552 \\
4 & 0.7320 & 0.7300 \\
5 & 0.7517 & 0.7478 \\
6 & 0.8890 & 0.8739 \\
7 & 1.4817 & 1.4730 \\
8 & 2.2449 & 1.7379 \\
9 & 2.4042 & 1.8821 \\
10 & 2.5298 & 2.2938 \\
\hline
\end{tabular}




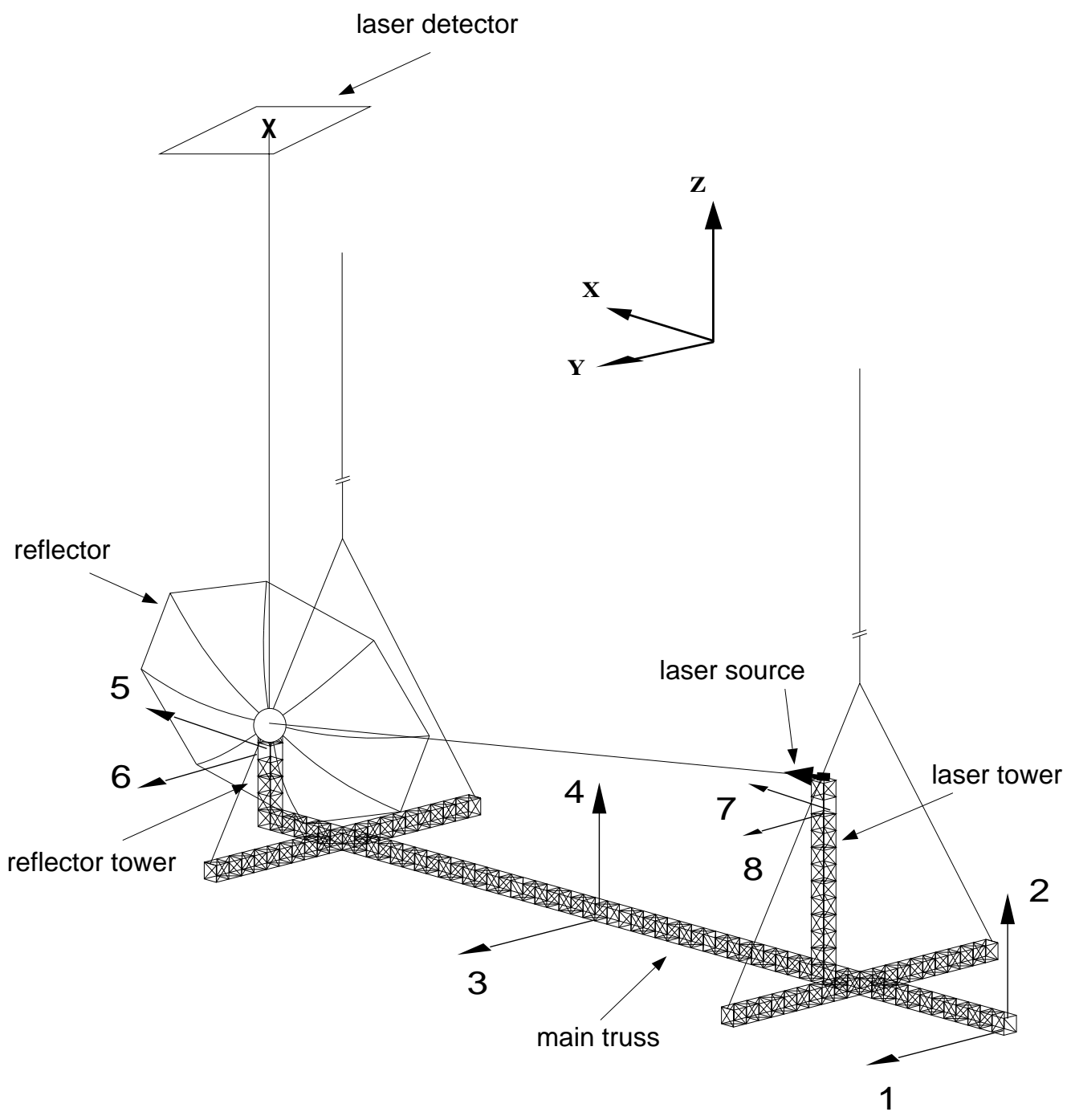

Fig. 1 Schematic of the CEM testbed. 


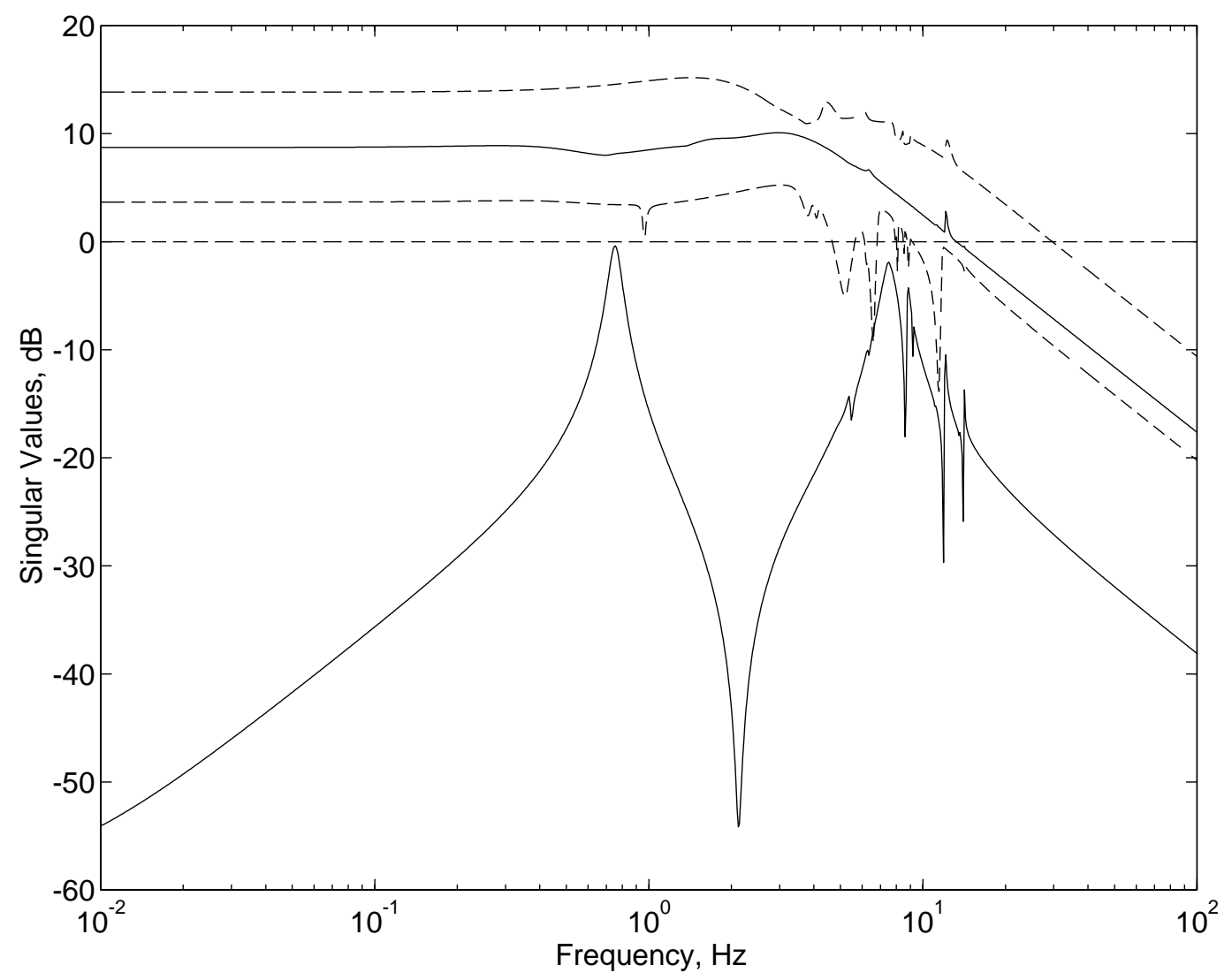

Fig. 2 Maximum and minimum singular value plots of $L Q G$ dissipative controllers for phase-0 CEM (dashed line) and phase-1 CEM (solid line). 
Fig. 3 CEM testbed. 


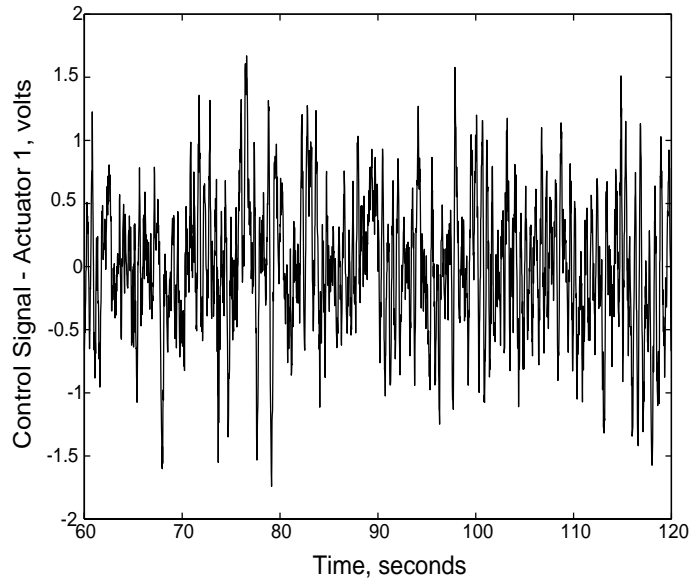

(a)

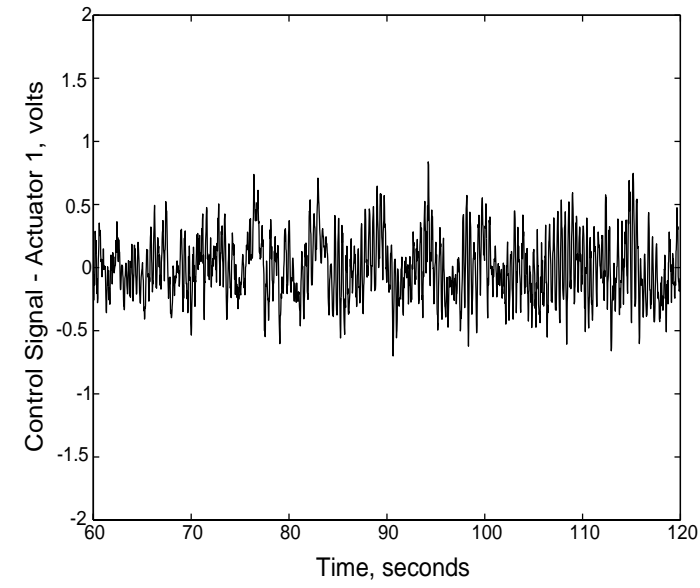

(b)

Fig. 4 Control signals at station 3, with static dissipative controller, (a) phase-0 CEM: and (b) phase-1 CEM. 


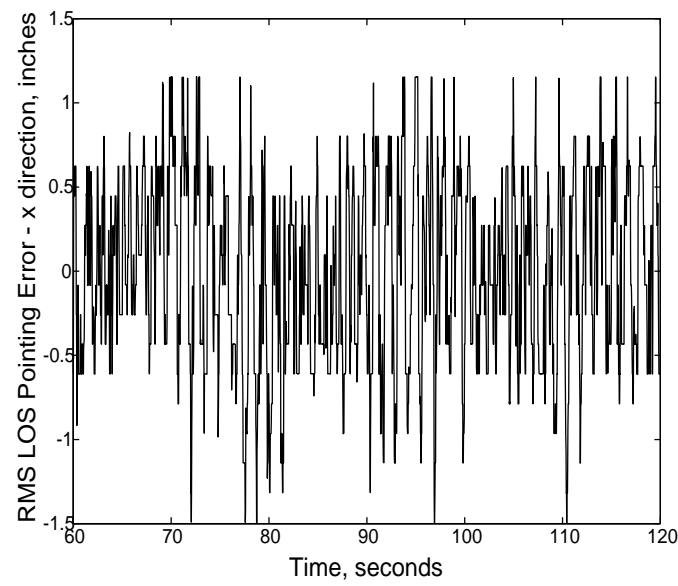

(a)

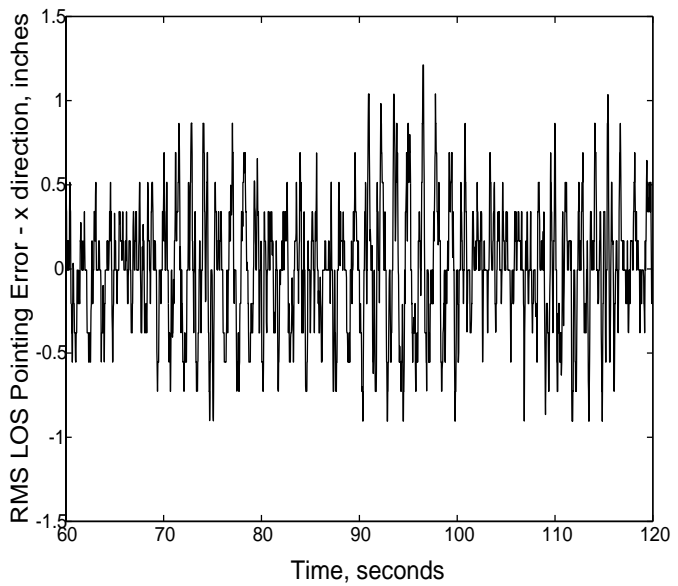

(b)

Fig. 5 Line-of-sight pointing error in x-direction, with static dissipative controller: (a) phase-0 CEM, and (b) phase-1 CEM. 


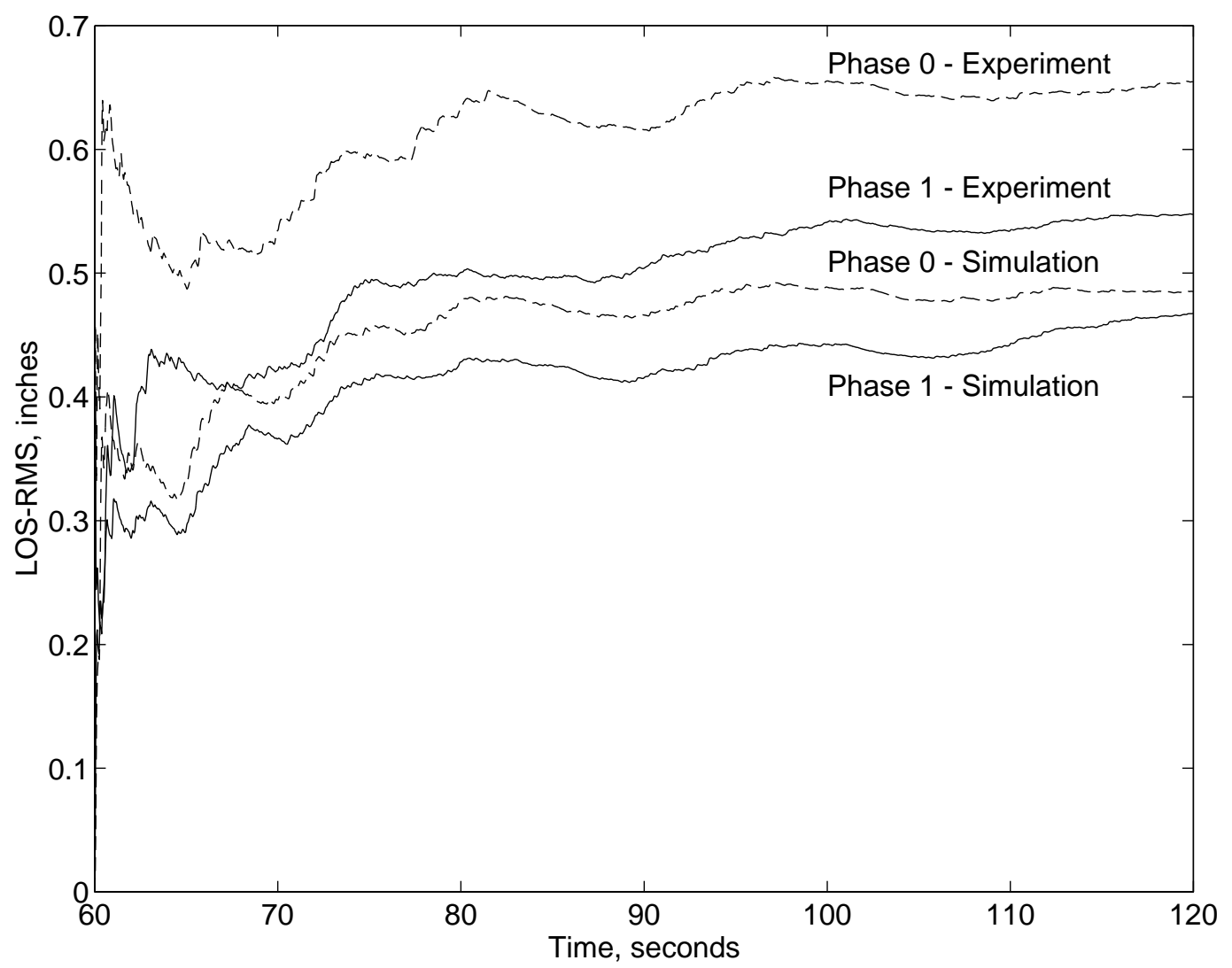

Fig. 6 Comparison of rms line-of-sight pointing error, with Static Dissipative Controllers. 


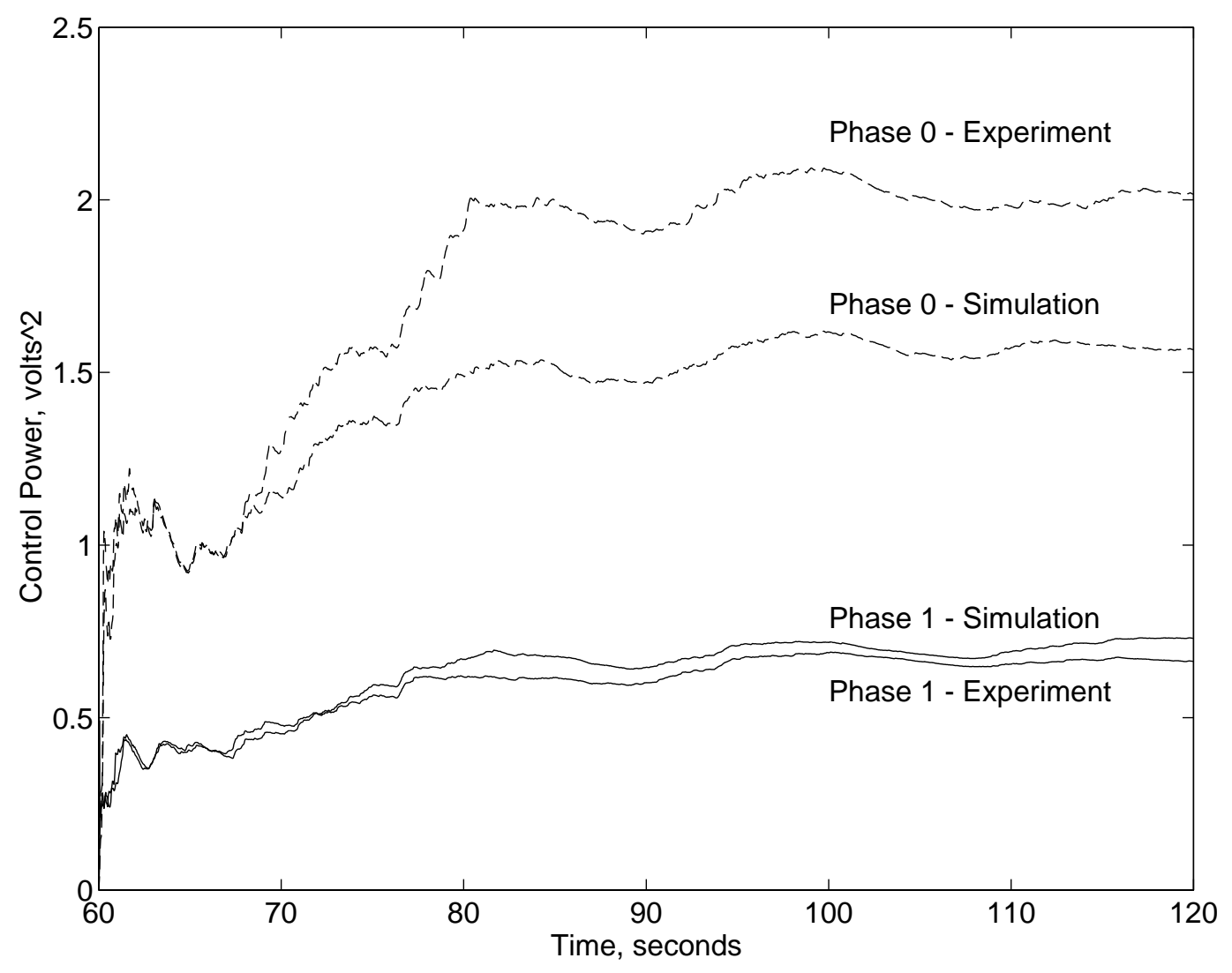

Fig. 7 Comparison of average control power, with static dissipative controllers. 


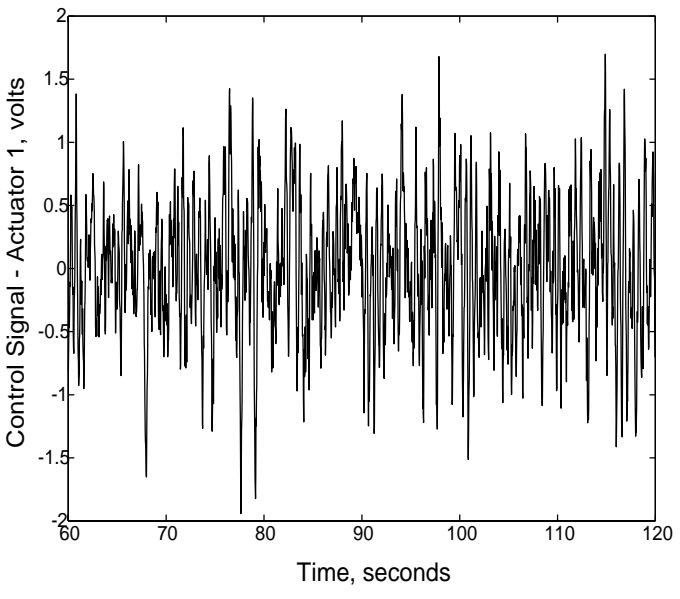

(a)

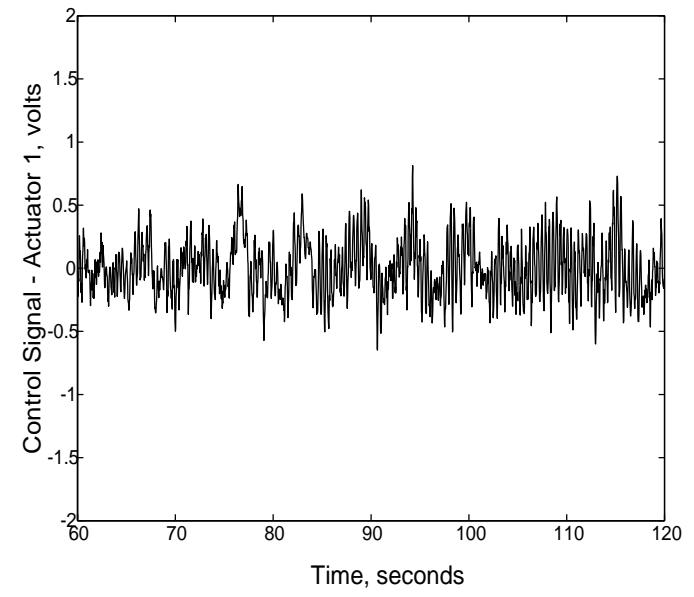

(b)

Fig. 8 Control signals at station 3 , with static dissipative controller: (a) phase-0 CEM and (b) phase-1 CEM. 


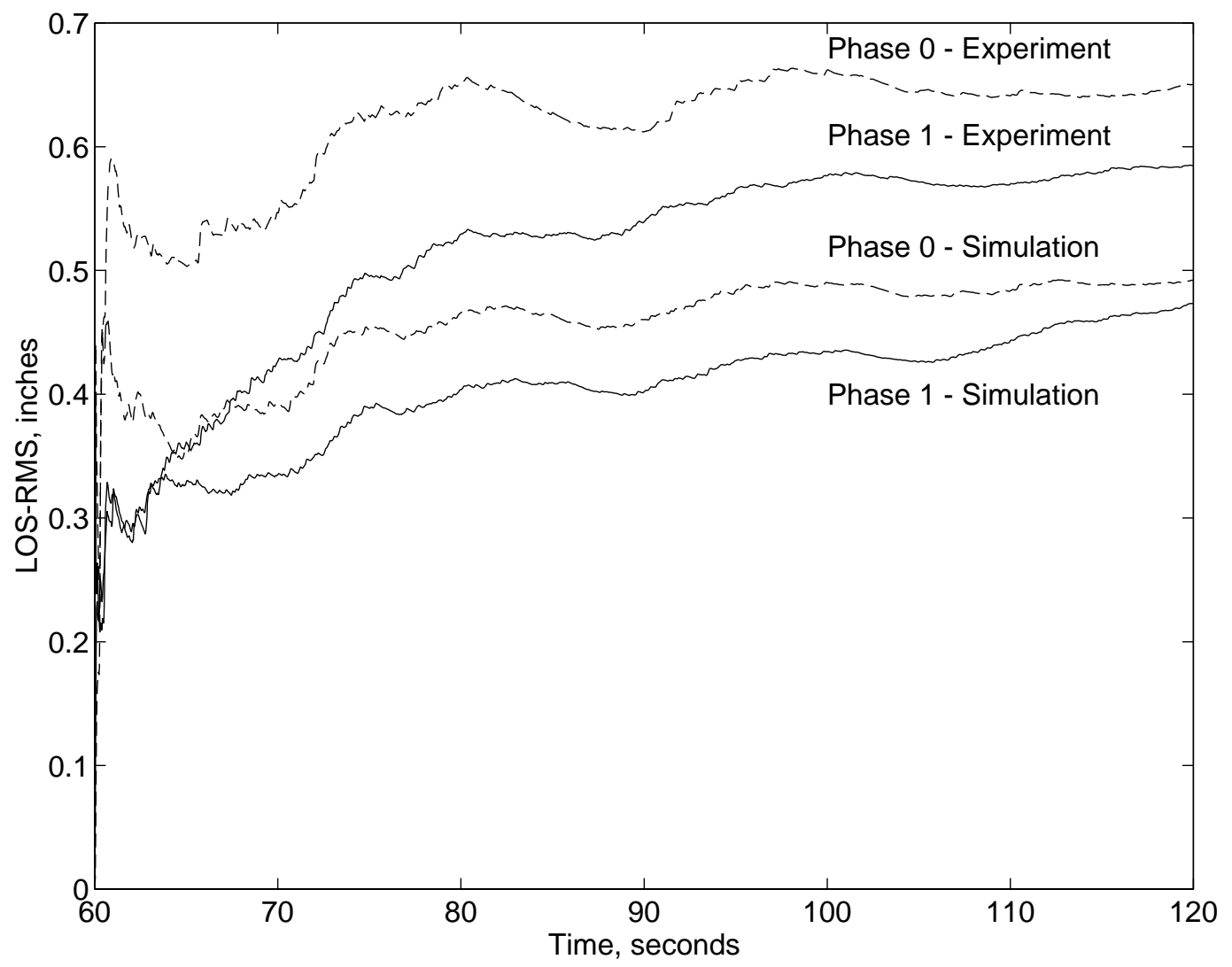

Fig. 9 Comparison of rms line-of-sight pointing error, with dynamic dissipative controllers. 


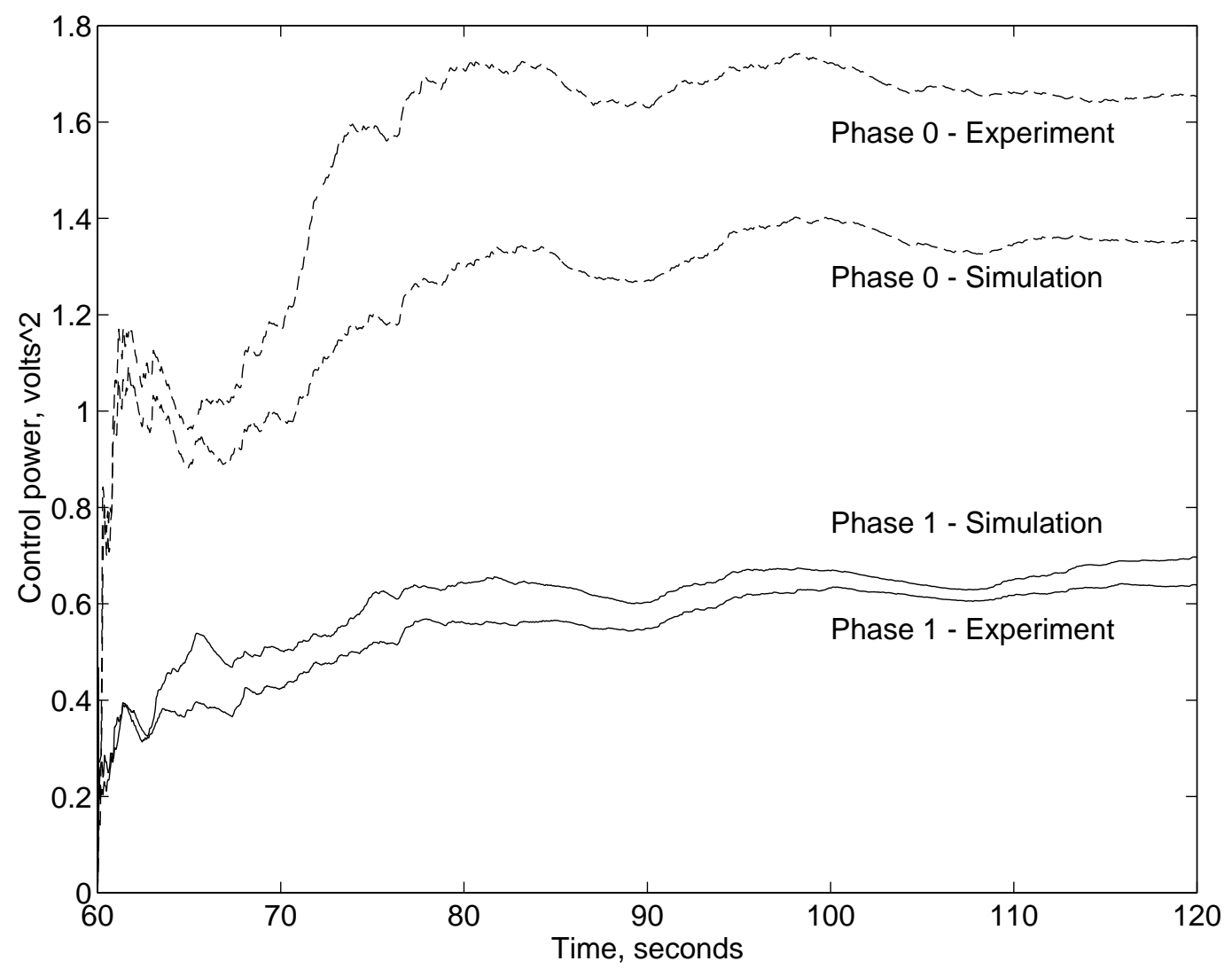

Fig. 10 Comparison of average control power, with dynamic dissipative controllers. 


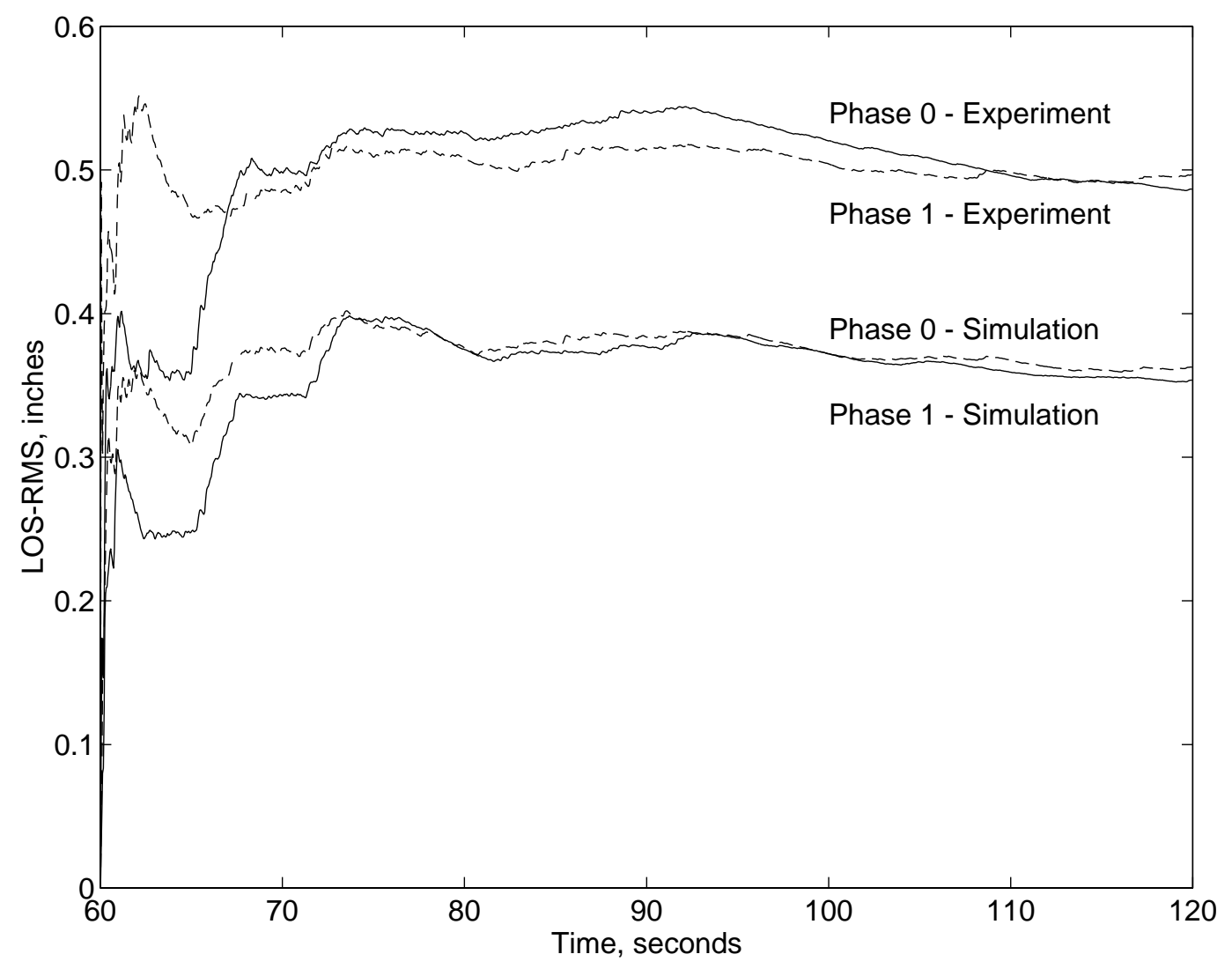

Fig. 11 Comparison of rms line-of-sight pointing error, with LQG dissipative controllers. 


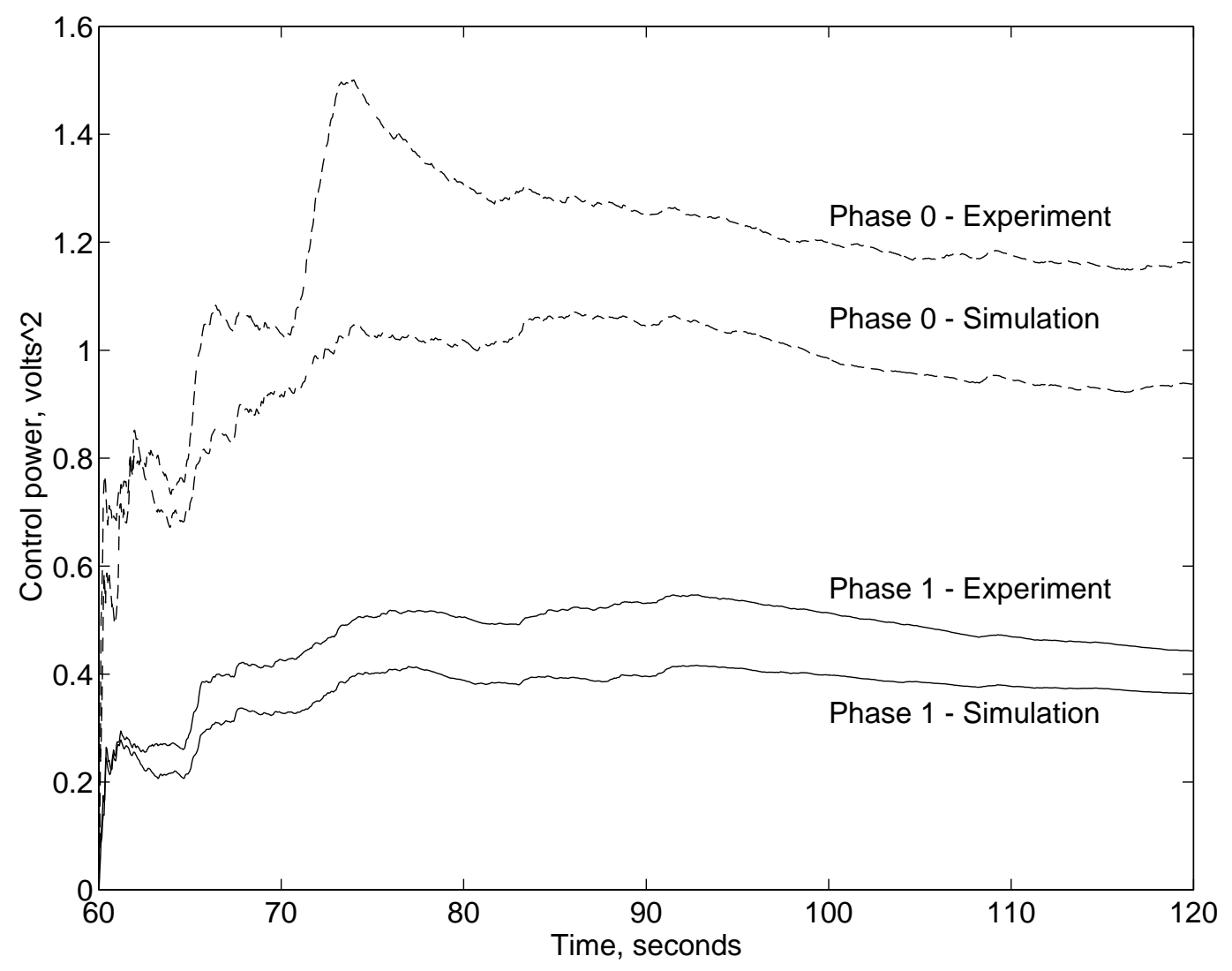

Fig. 12 Comparison of average control power, with LQG dissipative controllers. 